\title{
Knowledge, Attitude, Practice and associated factors on menstrual hygiene among high school students of North Wollo Zone, Ethiopia, 2019: A cross-sectional study
}

Getnet Gedefaw ( $\sim$ gedefawget@gmail.com )

Woldia University https://orcid.org/0000-0003-4906-2555

Fentanesh Endalew

Woldia University

Bitewsh Azmeraw

Woldia University

Bethelhem Walelign

Woldia University

Eyob Shitie

Woldia University

\section{Research note}

Keywords: Menstrual hygiene, knowledge, high school students, Ethiopia

Posted Date: August 12th, 2019

DOI: https://doi.org/10.21203/rs.2.12618/v1

License: (c) (i) This work is licensed under a Creative Commons Attribution 4.0 International License.

Read Full License 


\section{Abstract}

Objective Lack of proper menstrual hygiene predisposes women to different infectious and chronic obstetric and gynecological problems. A Facility based cross-sectional study was implemented from October 1-December 10, 2018. The data was collected using structured, pretested questioners among female high school students. Systematic random sampling technique was implemented. Data was entered and analyzed through Epi data 3.1 and SPSS respectively. Therefore, this study aimed to assess knowledge, attitude, practice and its associated factors of menstrual hygiene among high school students in North Wollo Zone, Woldia, Ethiopia. Results This study revealed that 365(89.2\%), 200(48.9\%) and $196(47.9 \%)$ of the study participants have good knowledge, good practice and good attitude about menstrual hygiene respectively. Being grade 10 students [AOR=3.96, 95\% $\mathrm{Cl}=2.0-7.8$ ], and having good practice of menstrual hygiene ( $\mathrm{AOR}=2.52,95 \% \mathrm{Cl}=1.26-5)$ had positive association with menstrual hygiene knowledge. Maternal education level ( $A O R=1.86,95 \% \mathrm{Cl}=1.18-2.9)$, being grade 10 students (AOR $=2.3,95 \% \mathrm{Cl}=1.48-3.56)$ were associated factors for practicing menstrual hygiene. Being grade 10 students $(A O R=1.9,95 \% \mathrm{Cl}=1.2-2.8)$, age $\geq 18$ years $(A O R=1.67,95 \% \mathrm{Cl}=1.09-2.55)$ were statistically and positively associated with the attitude of menstrual hygiene.

\section{Introduction}

Menstrual hygiene is personal hygiene during menstrual flow which includes bathing once a day, changing clothes regularly, and changing pads at least two- four times per day (1,2). In developing country menstrual hygiene is a problem for adolescent girls, particularly when attending schools (3). During menstruation, girls have been facing both practical and strategic gender problems. These have negative impacts for their personal lives and development opportunities (4). Reproductive age women who had not well hygienic practices are also prone to infertility. The effect of poor menstrual hygiene management, however, remains unclear even though menstrual hygiene management can affect the reproductive tract with unclear route of transmission (5).

Menstrual hygiene is an issue that is insufficiently acknowledged and has not received adequate attention in the reproductive health as well as water, sanitation, and hygiene (WASH) sectors in developing countries $(6,7)$. Menstrual hygiene is an issue that every woman of reproductive age is faced with in Ethiopia (8). Lack of knowledge and poor personal hygienic practices during menstruation can lead to various gynecological problems in the reproductive life of girls. Therefore to manage menstrual complications, essential reproductive health services and youth friendly services should be well addressed including access to water and sanitation $(9,10)$. There is also a need for both men and women to have a greater awareness of good menstrual hygiene practices (11). Studies are too limited in Ethiopia regarding knowledge, practice, and attitude towards menstrual hygiene. Therefore, this study helps to identify gaps relevant to attitude, practices, environmental, social and health factors and challenges of menstrual hygiene among high school students.

\section{Methods}




\section{Study setting}

Woldia is the capital city of North Wollo zone, Amhara region which is situated $521 \mathrm{~km}$ from Addis Ababa, the capital city of Ethiopia. Woldia town has one preparatory school and three high schools (selam, millinium, and woldia general high school). The town has four rural and three urban kebelle. In these high schools, there are about 7,027 (3217 in Woldia high school, 1500 in Wergiesa high school and 2310) in Mekiet high school.

\section{Study design}

An institution-based cross-sectional study was implemented from October 1- December 10, 2018

\section{Source population}

All female students in the selected North wollo high schools

\section{Study population}

Female students who have had menarche in selected high schools during the data collection time were included

\section{Sample size determination and sampling procedure}

The sample size was determined by using Epi info 7software Stat Cal with the assumptions of single population proportion formula.

The actual sample size is calculated using single population proportion formula.

$$
\begin{aligned}
& \mathrm{n}=\left(\underline{\mathrm{Z}}_{\mathrm{a} / 2}\right)^{2} \underline{\underline{2}} \mathrm{p}(\underline{1-\mathrm{P}})=(\underline{1.96}) \underline{\underline{2}} .0 .513(\underline{1-0.513})=384 \\
& d^{2} \quad(0.05)^{2}
\end{aligned}
$$

Where: $-\mathrm{n}=$ minimum sample size required for the study

$Z=$ standard normal distribution $(Z=1.96)$ with confidence interval of $95 \%$

$\mathbf{P}=$ knowledge of girls on menstrual hygiene in Ethiopia $(p=51.36 \%)[17]$.

$\mathrm{d}=$ is a tolerable margin of error $(\mathrm{d}=0.05)$

The final sample size was come up by adding a non-response rate of $10 \%$ to the sample size from 384 . Therefore, the final sample size for this study was $\mathbf{4 2 3 .}$

The systematic random sampling technique was applied to select Woldia high school, millennium high school and selam high school from woldia town where as Filakit and wurgesa high schools were selected from Mekiet and Habru woreda respectively. 


\section{Data Collection tools}

The data were collected using structured, pretested questioners from the selected female students, using a questionnaire adapted from relevant literature of similar studies. The questionnaires consists of basic socio-demographic characteristics, knowledge on menstrual hygiene, attitude on menstrual hygiene, and practice of menstrual hygiene

\section{Operational definition}

Good Knowledge: Study participants who scored below the mean score of the respondents

Poor knowledge: Study participants who scored below the mean score of the respondents

Good Practice: Study participants who scored the mean score of the respondents

Poor practice: Study participants who scored below the mean score of the respondents

Good attitude: Study participants who scored the mean score of the respondents

Poor attitude: Study participants who scored below the mean score of the respondents

\section{Data processing and analysis}

Completeness of the questionnaire was rechecked then the data were coded, entered and analyzed using Epi Data3.1 and SPSS Version 23 respectively. Variables in the bivariate model, those variables with $\mathbf{p}<0.2$ were a candidate to multivariable logistic regression analysis so as to control confounding factors in the association. Finally, P-value of $<\mathbf{0 . 0 5}$ was used to declare the statistical significance of the variables.

\section{Ethical consideration}

Ethical clearance was obtained from Woldia university ethical clearance committee and formal letter have been submitted to each high schools to get permission. School directors, unit leaders, and school supervisors were briefed on the objective of the study. Informed consent for each participant has obtained. Confidentiality and privacy of participants were secured.

\section{Results}

\section{Socio-demographic factors of the study participants}

Among 423 of the study participants, 409 of them have provided valuable information with a response rate of $96.7 \%$. The mean age of the study participants was 16.4 years (ranged from 14- 20 years) with SD of \pm 1.5 years. Regarding the age of the study participants, more than half of them $211(51.6 \%)$ was within the range of 16-17 years. (table 1 ). 


\section{Knowledge on menstrual hygiene}

Out of 409 study participants, 365(89.2\%) of them had good knowledge about menstruation and its hygiene. About $361(88.3 \%)$ of the participants reported that poor hygiene during menstruation may cause diseases and $298(72.9 \%)$ of them reported that having good hygiene during menstruation may reduce diseases concurrence due to poor hygiene during menstruation (table 2).

\section{Practice on menstrual hygiene}

From the total study participants, $200(48.9 \%)$ of them had good menstruation hygiene practice. Nearly all of the respondents $356(87 \%)$ of them had reported that the type of absorbent that they have been used during menstruation was sanitary pad (table 3 ).

\section{Attitude on menstrual hygiene}

Among 409 study participants, 196(47.9\%) of them had good attitude about menstrual hygiene. Among the total respondents, $354(86.6 \%)$ of them had reported that menstruation is good for health(table 4 ).

\section{Factors associated with knowledge on menstrual hygiene}

There were three explanatory variables in a bivariate analysis which had $\mathbf{p}<0.2$ : grade level, fathers' level of occupation, and practice of the participants.

In the multivariable analysis: students grade level and practice of the students were statistically associated with menstrual hygiene knowledge $(\mathbf{p}<0.05)$ via backward stepwise regression.

Girls who were grade 10 student's had 3.96 times more likely to have good knowledge as compared to girls who were grade 9 students [AOR=3.96, 95\%Cl $=2-7.8]$.

Students who had good menstrual practice had 2.52 times more likely to have good knowledge on menstrual hygiene as compared to those who had poor practice on menstrual hygiene ( $A O R=2.52,95 \%$ $=\mathrm{Cl}(1.26-5)$ (table 3$)$

\section{Factors associated with practice on menstrual hygiene}

There were three explanatory variables in a bivariate analysis which had $p-<0.2$ : grade level, mother's level of education, and knowledge participants.

In the multivariable analysis: students grade level, higher level of maternal education and knowledge of the students were significantly associated $(p<0.05)$ using backward stepwise regression

Girls who had good knowledge on menstrual hygiene have 3.74 more likely good practices on menstrual hygiene as compared to Girls who had poor knowledge on menstrual hygiene (AOR=3.74,95\% $\mathrm{Cl}(1.18$ -

7.7). 
Respondents whose mothers level of education is secondary and above had 1.86 more likely good practice as compared with those whose mothers level of education is elementary and no formal education(AOR=1.86,95\% $\mathrm{Cl}(1.18-2.9)$

Being a grade 10 students had 2.3 more likely had good practice on menstrual hygiene as compared to grade 9 students(AOR $=2.3,95 \% \mathrm{Cl}(1.48-3.56)$ (table 3$)$.

\section{Factors associated with attitude on menstrual hygiene}

There were two explanatory variables in the bivariate analysis which had $\mathrm{p}<0.2$ : grade level, and age of the participants.

In the multivariable analysis: students grade level and age of the students were statistically associated $(p<0.05)$ through backward stepwise regression

Grade 10 respondents 1.9 more likely good attitude on menstrual hygiene as compared with grade 9 respondents (AOR=1.9, 95\% $\mathrm{Cl}(1.2-2.8)$.

Students whose age was $>=18$ had 1.67 more likely good attitude on menstrual hygiene as compared with 13-14 and 16-17 age group respondents(AOR=1.67(1.09-2.55) (table 3).

\section{Discussion}

This study revealed that $89.2 \%, 48.9 \%$ and $47.9 \%$ of study participants had good knowledge, good practice and good attitude with $95 \% \mathrm{Cl}(86.1-92.2), 95 \% \mathrm{Cl}(44.3-53.5)$ and $95 \% \mathrm{Cl} 42.6-53.3)$ respectively.

Regarding the knowledge of menstruation, this study finding is in line with the study done in America (15). The possible reason may be due to both the study was conducted in high school students. This study finding is higher than the study conducted in Ethiopia (Nekemte), Nigeria; Ethiopia (Amhara), India and Saudi Arabia $(14,3,13,1217)$. This could be due to the number of study participants and the study site in each study.

Regarding the practice of menstruation, the finding of this study is higher than the study done in Ethiopia (Wogera), and Nigeria $(2,3)$. The discrepancy might be due to this study only incorporates secondary school students, unlike other studies.

The finding of this study is also lower than the study conducted in America (15). This might be due to the fact that in developing countries there is low reproductive health coverage than developed countries and also the socioeconomic capacity of the developing countries has a direct impact on the utilization of reproductive health services.

This study finding is higher than the study implemented in western Iran (16). This could be due to the report from Iran showed that the study is done in all school girls. 
Regarding the attitude of menstruation, this study finding is lower than the study implemented in western Iran. This could be due to in this study the sample size as small as compared to the study done in western Iran (16).

Good knowledge is one of the associated risk factor for menstrual hygiene practice. A similar finding is reported in Gondar (13). This could be due to the fact that being knowledgeable has a direct impact to practice menstrual hygiene management.

Being a grade 10 student is another important associated risk factor for menstrual hygiene practice. This is because when students are learning more about menstruation and its hygiene they can easily understand the future risk and complications of poor practicing of menstrual hygiene management.

Good practicing of menstrual hygiene was associated with the knowledge of menstrual hygiene. This might be due to the fact that when students having good menstrual hygiene practice they have good menstrual knowledge before.

Being a grade 10 student is another important associated risk factor for knowledge of menstrual hygiene. This might be due to the fact that grade 10 students' may have more exposure to some reproductive and youth friendly services that makes aware them about menstrual hygiene in their schools.

The age of the students within the range of above 18 is associated with the attitude of menstrual hygiene. This could be due to the fact that when their age increases their exposure to reliable information about menstrual hygiene practice.

A grade 10 student and age>18are associated factor for the attitude of menstrual hygiene. This is due to the fact that as the age and their grade level increases, the mentality is also increased and exposure to information about menstrual hygiene is also increased.

\section{Conclusion}

This study revealed that the knowledge of the study participants towards menstrual hygiene was high whereas attitude and practice regarding menstrual hygiene was low. Being grade 10 students and having good practice were the associated factors of knowledge. Higher level maternal of education, being grade 10 students and having good knowledge towards menstrual hygiene were the identified associated factors for implementing of menstrual hygiene practice. Being grade 10 students and age $\geq 18$ were statistically positively associated with attitude of menstrual hygiene. Therefore, creating awareness to reproductive health groups, educating their families and parents can change their poor practicing and attitude of menstrual hygiene.

\section{Limitation}

Risky behavioral history as well as using qualitative methods to gain deeper insights was not assessed. 


\section{Abbreviations}

AOR: Adjusted odd ratio, COR: Crude odd ratio, Cl: Confidence interval.

\section{Declarations}

\section{Acknowledgment}

The authors would like to thank the data collectors and supervisors for their invaluable and timely fashion work. The author's deep gratitude also goes to the study participants, school directors, supervisors and unit leaders who were volunteered and took their time to give us all the relevant information for the study.

\section{Funding}

Not applicable

\section{Availability of data and materials}

All the datasets used and analyzed during the current study data and its supplementary files are available from the corresponding author on reasonable request.

\section{Author's Contributions}

FE, ES, BW, and AZ have initiated the research, wrote the research proposal, conducted the field work, supervised data entry, analyzed the data and wrote the manuscript. GG and FE participated in refining the research proposal, analyzed the data and wrote the report. All authors read and approved the final manuscript.

\section{Ethics approval and consent to participate}

Ethical approval was obtained from the Faculty of health science, Woldia university research and community service office. All the study participants were informed about the importance and the objective of the study. Written consent was obtained from the partner or legal guardians for those women aged less than 18 years. Moreover, assent was secured from participants aged below 18 years. Students whose age were 18 years and above provided by written consent on their own

\section{Consent to publish}

Not applicable.

\section{Competing interest}

The authors declared that they have no competing interests. 


\section{Funding}

Woldia University. The funder has no role in the design of the study, collection, analysis, and interpretation of data and in writing the manuscript.

\section{References}

1. Warenius L, Pettersson KO, Nissen E, Hojer B, Chishimba P, Faxelid E. Vulnerability and sexual and reproductive health among Zambian secondary school students. Culture, health \& sexuality. 2007 Sep-Oct;9(5):533-44. PubMed PMID: 17687677. Epub 2007/08/10. eng.

2. Meseret Abay Fisseha, Yigzaw Kebede, Hedija Yenus Yeshita. Menstrual Hygiene Practice and Associated Factors among Secondary School Girls in Wegera District, Northwest Ethiopia; a CrossSectional Study. Computational Biology and Bioinformatics. Vol. 5, No. 1, 2017, pp. 6-11. doi: 10.11648/j.cbb.20170501.12

3. Fehintola FO et al: Assessment of knowledge attitude and practice about menstruation and menstrual hygiene among secondary school girls in Ogbomo, Oya state, Nigeria. Int J Reprod Contracept Obstet Gynecol. 2017 May;6(5):1726-1732

4. UNICEF: Menstrual Hygiene Management in Schools: Applied Learning and Improved Practice. A Multi-Setting Approach for Sierra Leone - Country Report, 2012.

5. Sumpter $C$, Torondel B. A systematic review of the health and social effects of menstrual hygiene management. PLoS One. 2013;8(4):e62004. Published 2013 Apr 26. doi:10.1371/journal.pone.0062004

6. Balamurugan, et al.: Study on menstrual hygiene among reproductive age group women. Journal of Basic and Clinical Reproductive Sciences · July - December $2014 \cdot$ Vol $3 \cdot$ Issue 2

7. Eleen Korir et al. : menstrual hygiene management among adolescent girls in primary school in mashuru division. The Pan African Medical Journal. 2018;31:222. doi:10.11604/pamj.2018.31.222.13521

8. Kuhlmann AS, Henry K, Wall LL. Menstrual Hygiene Management in Resource-Poor Countries. Obstet Gynecol Surv. 2017;72(6):356-376. doi:10.1097/OGX.0000000000000443.

9. Kansal S, Singh S, Kumar A. Menstrual hygiene practices in context of schooling: A community study among rural adolescent girls in Varanasi. Indian J Community Med 2016;41:39-44.

10. Dissertation on the effect of school based health education regarding menstrual hygiene -an intervention study among adolescent girls of perambalur district, 2018

11. Sustainable Sanitation Alliance (SuSanA). London School of Hygiene and Tropical Medicine Barriers and facilitators of school-based menstrual hygiene promotion in Cambodia: policy, implementation and water, sanitation and hygiene (WASH) school environment, june, 2018.

12. Ramachandra $\mathrm{K}$ et al.: knowledge and practices regarding menstrual hygiene among urban adolescent girls, in Bangalore, India, Int J Contemp Pediatr. 2016Feb;3(1):142-145. 
13. Uzochukwu Uzoma Aniebue et al. Impact of pre-menarcheal training on menstrual practices hygiene of Nigerian school girls. Pan African journal. Vol 1, No 2, 2009

14. Upashe SP, Tekelab T, Mekonnen J. Assessment of knowledge and practice of menstrual hygiene among high school girls in Western Ethiopia. BMC women's health. 2015 Oct 14;15:84. PubMed PMID: 26466992. Pubmed Central PMCID: PMC4606849. Epub 2015/10/16. eng.

15. Washinton DC U.S. Grace Meng Renews Effort to Make Menstrual Hygiene Products More Accessible and Affordable to Women, Feb, 2017.

16. S Mohammad-Alizadeh et al. Practice of Iranian Adolescent Girls Regarding Puberty and Menstrual Hygiene and its Predictors. Int J Women's Health Reproduction Sci Vol. 2, No. 3, 2014

17. Moawed S. Indigenous practices of Saudi girls in Riyadh during their menstrual period. Eastern Mediterranean health journal $=$ La revue de sante de la Mediterranee orientale $=$ al-Majallah alsihhiyah li-sharq al-mutawassit. 2001 Jan-Mar;7(1-2):197-203. PubMed PMID: 12596970. Epub 2003/02/25. eng.

\section{Tables}

Due to technical limitations, tables are only available as a download in the supplemental files section.

\section{Supplementary Files}

This is a list of supplementary files associated with this preprint. Click to download.

- Tables.pdf 